\title{
Potential Novel Prediction of TMJ-OA: MiR-140-5p Regulates Inflammation Through Smad/TGF- $\beta$ Signaling
}

\author{
Weihao Li" ${ }^{*}$, Shurong Zhao', Hefeng Yang ${ }^{1}$, Chao Zhang ${ }^{2}$, Qiang Kang ${ }^{3}$, Jie Deng ${ }^{4}$, \\ Yanhua $X u^{1}$, Yu Ding ${ }^{1}$ and Song $L^{1{ }^{1 *}}$
}

${ }^{1}$ Department of Dental Research, School of Stomatology, Kunming Medical University, Kunming, China, ${ }^{2}$ School of Public Health, Kunming Medical University, Kunming, China, ${ }^{3}$ Department of Hepatobiliary Surgery, The Second Affiliated Hospital of Kunming Medical University, Kunming, China, ${ }^{4}$ Department of Oral Biology and Pathology, School of Dental Medicine Stony Brook, NY, United States

\section{OPEN ACCESS}

Edited by:

Mohamed M. Abdel-Daim,

Suez Canal University, Egypt

Reviewed by:

Agnieszka Barbara Najda, University of Life Sciences in Lublin,

Poland

Naoto Hirose,

Hiroshima University, Japan

*Correspondence:

Weihao L

121233015@qq.com

Song Li

lisong59@sohu.com

Specialty section

This article was submitted to Inflammation Pharmacology,

a section of the journal

Frontiers in Pharmacology

Received: 02 November 2018

Accepted: 07 January 2019

Published: 23 January 2019

Citation:

Li W, Zhao S, Yang H, Zhang C,

Kang $Q$, Deng J, Xu Y, Ding $Y$ and Li S (2019) Potential Novel Prediction of TMJ-OA: MiR-140-5p Regulates Inflammation Through Smad/TGF- $\beta$ Signaling. Front. Pharmacol. 10:15. doi: 10.3389/fphar.2019.00015
Temporomandibular joint osteoarthritis (TMJ-OA), mainly exhibit extracellular matrix loss and condylar cartilage degradation, is the most common chronic and degenerative maxillofacial osteoarthritis; however, no efficient therapy for TMJ-OA exists due to the poor understanding of its pathological progression. MicroRNA (miR)-140-5p is a novel non-coding microRNAs (miRNAs) that expressed in osteoarthritis specifically. To investigate the molecular mechanisms of miR-140-5p in TMJ-OA, primary mandibular condylar chondrocytes (MCCs) from C57BL/6N mice were treated with interleukins (IL)$1 \beta$ or transfected with miR-140-5p mimics or inhibitors, respectively. The expression of matrix metallopeptidase (MMP)-13, miR-140-5p, nuclear factor (NF)-kB, Smad3 and transforming growth factor (TGF)- $\beta 3$ were examined by western blotting or quantitative reverse-transcription polymerase chain reaction (qRT-PCR). The interaction between the potential binding sequence of miR-140-5p and the $3^{\prime}$-untranslated region ( $3^{\prime} \cup T R$ ) of Smad3 mRNA was testified by dual-luciferase assay. Small Interfering RNA of Smad3 (Si-Smad3) was utilized to further identify the role of Smad3 mediated by miR-140-5p. The data showed MMP13, miR-140-5p and NF-kB increased significantly in response to IL-1 $\beta$ inflammatory response in MCCs, meanwhile, Smad3 and TGF- $\beta 3$ reduced markedly. Moreover, transfection of miR-140-5p mimics significantly suppressed the expression of Smad3 and TGF- $\beta 3$ in MCCs, while miR-140-5p inhibitors acted in a converse manner. As the luciferase reporter of Smad3 mRNA observed active interaction with miR-140-5p, Smad3 was identified as a direct target of miR-140$5 \mathrm{p}$. Additionally, the expression of TGF- $\beta 3$ was regulated upon the activation of Smad3. Together, these data suggested that miR-140-5p may play a role in regulating mandibular condylar cartilage homeostasis and potentially serve as a novel prognostic factor of TMJ-OA-like pathology.

Keywords: mirna-140-5p, TMJ-OA, inflammation, TGF- $\beta$, Smad

Abbreviations: ANOVA, analysis of variance; MCCs, mandibular condylar chondrocytes; MMP13, matrix metallopeptidase 13; NF-kB, Nuclear factor kB; PBS, phosphate-buffered saline; PVDF, polyvinylidene difluoride; qRT-PCR, quantitative reverse-transcription polymerase chain reaction; SDS-PAGE, sodium dodecyl sulfate polyacrylamide gel electrophoresis; Smad2/3, Sma-and Mad-related protein $2 / 3$; TGF- $\beta 3$, transforming growth factor- $\beta$ 3; TMJ-OA, temporomandibular joint osteoarthritis. 


\section{INTRODUCTION}

Temporomandibular joint osteoarthritis is a common condition in temporomandibular joint disorder (TMD). Approximately $40 \%$ of TMD patients presented TMJ-OA (Paniagua et al., 2017). Mainly exhibit extracellular matrix loss, condylar cartilage degradation and synovial inflammation, TMJ-OA patients often accompanied with X-ray signs in clinic (Zhao et al., 2011). TMJ-OA usually accompanied by occlusal disorder which affects the patients' life severely (Cordray, 2016). As secondary cartilage, mandibular condylar cartilage differs from other articular cartilage in certain aspects. Composed of fibrocartilage, mandibular condylar cartilage contained several thick multilayers of collagen fiber zones while other articular cartilage mainly consists of hyaline cartilage (Bouvier and Zimny, 1987). Dysregulation of chondrocyte catabolism is the key in the pathologic process of OA (Zhang et al., 2016). Previous studies reported that highly expression of type $\mathrm{X}$ collagen, thickening hypertrophic layer, and bone remodeling were related to the pathological alterations of TMJ-OA (Goldring, 2012; Hashimoto et al., 2013; Zhang et al., 2013; Duan et al., 2015; Zhang J. et al., 2018). Moreover, mandibular condylar cartilage seems to be more sensitive to mechanical stress (Duan et al., 2015). In spite of previous investigations, the molecular mechanism of TMJ-OA are still poorly understood. What's more, no effective targeted therapy or early prediction for TMJ-OA exists.

Interleukins- $1 \beta$ is believed to be critical in cartilage damage because it can restrain matrix protein synthesis and induces proinflammatory cytokines (Kapoor et al., 2011). Previews study proved that IL-1 can cause a complex series of changes lead to synovitis, mesenchymal proliferation, and cartilage lesion. The rat OA model established by IL-1 $\beta$ in vitro showed lower levels of phosphorylated p38, phosphorylated JNK, and beta-catenin (Zeng et al., 2014). According to previous study results, IL-1 $\beta$ induced an efficient inflammatory response in cartilage, which resemble TMJ-OA-like change.

MicroRNA(miR/miRNAs) are novel non-coding RNAs that regulate target genes negatively. Approximately $1 / 3$ of human mRNAs can be regulated by miRNAs, which demonstrated that miRNAs played essential roles in controlling gene expression (Lewis et al., 2005). MiRNAs inhibit its target genes by complementary binding to the $3^{\prime}$-untranslated regions ( $3^{\prime}$ UTR) of mRNA (Liu et al., 2015). Many miRNAs have been implicated in tissue-specific or development stage-specific of various diseases, including heart disease (van Rooij et al., 2006), arthritis (Iliopoulos et al., 2008), osteoarthritis and bacterial virus infection (Liu et al., 2015). When compared with miR140-3p, miR-140-5p seems to play more important function in the BMG rat model (Min et al., 2015). Miyaki et al. (2009) reported that miR-140 expressed largely difference between human articular chondrocytes and mesenchymal stem cells, and the expression of miR-140 found reduced in human osteoarthritis cartilage. However, Swingler et al. (2012) reported that the expression of miR-140-5p and miR-455-3p was increased in OA cartilage when compared with the cartilage of femoral neck fractures patients. These findings demonstrated that abnormal gene expression of miR-140-5p may contribute to different stages of development in $\mathrm{OA}$, however, the exact regulatory mechanism of miR-140-5p is still unclear, especially in TMJ-OA.

Recent studies showed that the TGF- $\beta$ superfamily play essential roles in almost all aspect of development, from the generation of germ cells to organ formation, and into postnatal life (Wang et al., 2014). TGF- $\beta 3$ expressed highly in ribs and vertebral cartilage. Previews studies showed that TGF- $\beta 3$ serve an important role in cartilage maintenance and homeostasis (Sun et al., 2018). The TGF- $\beta$ family transfer signal transmission primarily be conducted via Smad proteins, which acting a role of receptor activation in signal transduction. Consequently, TGF$\beta /$ Smads regulate cartilage development, including chondrocytes proliferation, early differentiation, hypertrophy, terminal differentiation, and maintenance of homeostasis (Sun et al., 2016). Interestingly, no research has completely clarified the regulatory mechanism of microRNA in TMJ-OA. In the present study, we identified that Smad3 is the direct target of miR140-5p. Dysregulation of miR-140-5p contributes to TMJ-OA pathogenesis through Smad/TGF- $\beta$ signaling.

\section{MATERIALS AND METHODS}

\section{MCCs Preparation}

C57BL/6N mice (2-week-old, male, $5 \mathrm{~g}$ ) were obtained from Model Animal Research Center of Kunming Medical University (Yunnan, China). This study was carried out in accordance with the recommendations of Chinese Academy of Sciences. Mice mandibular condylar cartilage were cut into tiny fragments, then digested with $0.25 \%$ trypsin (Gibco Invirtrogen, United States) for $25 \mathrm{~min}$ at $37^{\circ} \mathrm{C}$ and $0.2 \%$ collagenase (Sigma-Aldrich, United States) for $45 \mathrm{~min}$ at $37^{\circ} \mathrm{C}$. Followed by dissociation and filtration, cells were centrifuged at $1200 \mathrm{rpm}$ for $5 \mathrm{~min}$. Primary MCCs were cultured according to previous method (Gosset et al., 2008). Freshly isolated at passage 1 (P1), MCCs were placed in monolayer culture in 6-well plates (for RNA and protein) with DMEM/F-12 (Gibco Invirtrogen, United States) medium containing 10\% fetal bovine serum (Gibco Invirtrogen, United States). After serum starved for $12 \mathrm{~h}$, MCCs were induced by $10 \mathrm{ng} / \mathrm{ml}$ IL-1 $\beta$ (R\&D systems, Minneapolis, United States) stimulation for $24 \mathrm{~h}$, then treated with or without transfection experiments, respectively. Every 3 days, medium in the 6-well plates was refreshed for once.

\section{MCCs Transfection}

miR-140-5p mimics, miR-140-5p inhibitors, and their corresponding negative controls were designed and obtained from RiboBio (RiboBio, Guangzhou, China). Cultured and plated in 6-well plates ( $\sim 50 \%$ confluence), MCCs were starved for $12 \mathrm{~h}$, followed by $48 \mathrm{~h}$ transfection of miR-140-5p mimics or inhibitors, then treated with IL- $1 \beta$ for $24 \mathrm{~h}$, respectively. MCCs transfected with microRNA mimics or inhibitors of miR140-5p at a concentration of $50 \mathrm{nM}$ by using riboFECTTM CP Transfection Kit (166T) (RiboBio, Guangzhou, China) according to the manufacturer's protocol. 


\section{Immunofluorescence Analysis}

Mandibular condylar chondrocytes were cultured on 18-mm glass coverslips (Thermo Fisher Scientific, United States) and stained for single immunofluorescence staining. Fixed in 50\% $(v / v)$ methanol/50\% $(v / v)$ acetone for $5 \mathrm{~min}$. Subsequently, cells were rinsed in $1 \times$ DPBS (Gibco Life Technologies, United States) for 3 times, each time $5 \mathrm{~min}$, followed by incubation with rabbit anti-Smad3 (1:200 dilution; Abcam, Cambridge, MA, United States) at $4^{\circ} \mathrm{C}$ overnight. FITClabeled goat anti-rabbit IgG (Beyotime, Shanghai, China) were used as secondary antibodies and incubate for $60 \mathrm{~min}$. Cell nucleus were counterstained with DAPI (Solarbio, Beijing, China) for $10 \mathrm{~min}$. Coverslips were mounted in fluorescent mounting medium (Solarbio, Beijing, China). Finally, the fluorescence micrograph were captured and analyzed by confocal laser scanning microscope system (Nikon, Co., Ltd., Tokyo, Japan).

\section{Dual-Luciferase Assay}

miRTarBase ${ }^{1}$ was utilized to predict the potential binding sequence between miR-140-5p and Smad3. The wild-type (WT) or mutant-type (MUT) $3^{\prime}$ URT of Smad3, which containing the predicted binding sequences or mutant binding sites, was cloned into pmiR-RB-REPORT ${ }^{\mathrm{TM}}$ luciferase vector (RiboBio, Guangzhou, China) by XhoI/NotI restriction sites, respectively. The coding sequences of $3^{\prime} \mathrm{UTR}$ of Smad3 for PCR-amplified primers were as follows: 5'-TATGTTGGCTGGAAAACCAC AA-3'. After amplification, the PCR products were cloned into the vector for dual-luciferase assay. At a concentration of $1.5 \times 10^{4}$, cells $(293 \mathrm{~T})$ in a 96-well plate were transfected with miR-140-5p or negative control by Lipofectamine ${ }^{\mathrm{TM}}$ 3000 (Invirtrogen, Carlsbad, CA, United States) and incubated for $48 \mathrm{~h}$. Finally, luciferase activity was measured posttransfection using Dual-Glo ${ }^{\circledR}$ Luciferase Assay System (Promega, Madison, WI, United States). The activity ratio of each group was normalized to the corresponding Renilla luciferase activity.

\section{SiRNA Smad3 Transfection}

Mandibular condylar chondrocytes were transfected with small interference RNA of mouse Smad3 (Si-Smad3) to suppress the Smad3 expression. According to the protocol, the transfection were performed by riboFECTTM CP Transfection Kit (166T) (RiboBio, Guangzhou, China). After starved for $12 \mathrm{~h}$, MCCs were followed by $48 \mathrm{~h}$ transfection of Si-Smad3.

\section{Quantitative Reverse-Transcription Polymerase Chain Reaction (qRT-PCR) Analysis}

Following to the manufacturer's instructions, $1 \mu \mathrm{g}$ for total RNA were extracted by Eastep ${ }^{\circledR}$ Super Total RNA Extraction Kit (Promega, Madison, WI, United States) then reversed transcribed into cDNA by A5000 GoScript ${ }^{\mathrm{TM}}$ Reverse Transcription System

${ }^{1}$ http://mirtarbase.mbc.nctu.edu.tw/php/index.php
(Promega, Madison, WI, United States). Then miRNAs were reverse transcribed into cDNA with stem-loop primers by using miDETECT A Track ${ }^{\mathrm{TM}}$ miRNA qRT-PCR Starter Kit (RiboBio, Guangzhou, China). According to the manufacturer's instructions, qRT-PCR was performed in a CFX96 system (Bio-Rad, Hercules, CA, United States) using SybrGreen qPCR Mastermix (DBI Bioscience, Ludwigshafen, Germany). $\beta$-actin and small nuclear RNA U6 were used as internal controls to normalized gene expressions. The thermal cycling condition was $95^{\circ} \mathrm{C}$ for $10 \mathrm{~min}$, followed by 40 cycles of $95^{\circ} \mathrm{C}$ for $30 \mathrm{~s}$ and $60^{\circ} \mathrm{C}$ for $30 \mathrm{~s}$. The relative expression of miR140-5p to U6, and relative expression of mRNA to $\beta$-actin were measured by using the comparative $2^{-\Delta \Delta c t}$ method (Livak and Schmittgen, 2001). Specific primers of mouse miR-140-5p and U6 were designed by RiboBio (RiboBio, Guangzhou, China). Primer sequences used for qRT-PCR were as follows: mouse $\beta$-actin [GenBank: NM_007393.5], forward $5^{\prime}$-GTGCTATGTTGCTCTAGACTTCG-3, reverse $5^{\prime}$ ATGCCACAGGATTCCATACC-3'; mouse MMP13 [GenBank: NM_007393.5]: sense $5^{\prime}$-CAGTTGACAGGCTCCGAGAA-3' and antisense $5^{\prime}$-CCACATCAGGCACTCCACAT- $3^{\prime}$.

\section{Western Blot Analysis}

Western blotting was performed as previously described (Zhang C. et al., 2018). MCCs were harvested by whole-cell lysates with ice-cold lysis buffer (1\% NP-40, $50 \mathrm{mM}$ Tris-HCL, $0.1 \%$ SDS, and $\mathrm{pH}$ 7.4 $150 \mathrm{mM} \mathrm{NaCl}$ ) and protease inhibitors (Beyotime, Shanghai, China). Normalized by BCA assay kit (Beyotime, Shanghai, China), proteins were separated by SDS-PAGE at $80 \mathrm{~V}$ for $30 \mathrm{~min}$, then $120 \mathrm{~V}$ for $1 \mathrm{~h}$. After proteins transferred, the PVDF membrane (Millipore, Billerica, MA, United States) was then blocked in $5 \%$ non-fat milk at $26^{\circ} \mathrm{C}$ for $1 \mathrm{~h}$. Next, the blocked membrane was hybridized with primary antibodies anti-Smad3 (1:5,000 dilution; Abcam, Cambridge, MA, United States), anti-TGF- $\beta 3$ (1:1,000 dilution; Abcam, Cambridge, MA, United States), anti-NF-kB (1:1,000 dilution; Abcam, Cambridge, MA, United States), anti-NF-kB2 (1:1000 dilution; Abcam, Cambridge, MA, United States) and anti$\beta$-actin (1:5,000 dilution; Abcam, Cambridge, MA, United States) at $4^{\circ} \mathrm{C}$ over night, followed by secondary antibodies goat anti-rabbit IgG (1:5,000 dilution; Abcam, Cambridge, MA, United States) at $26^{\circ} \mathrm{C}$ for $1 \mathrm{~h}$. Finally, the membrane was incubated with ECL Western blotting Kit (Beyotime, Shanghai, China). The immuno-reactivity percentages to control group was analyzed by Image $J$ or Image Lab software provided by the Institute of Stomatology.

\section{Statistical Analysis}

All data in the present study were represented as mean \pm standard deviation (SD). Each group was repeated three times $(n=3)$. GraphPad Prism 5.0 or SPSS 17.0 were performed to statistical analyses. Student's $t$-test was conducted when two groups compared. One-way ANOVA followed by Bonferroni post hoc tests was used for multiple groups comparisons. The statistically significant difference considered when $P$ values $<0.05$. 


\section{RESULTS}

\section{The Expression of MMP13, MiR-140-5p, NF- $\kappa$ B Increased but Smad3, TGF- $\beta 3$ Downregulated in Response to IL-1 $\beta$}

In order to established TMJ-OA model in vitro, MCCs were induced by IL-1 $\beta$ (10 ng/ml) for $24 \mathrm{~h}$. The effects of IL- $1 \beta$ were examined in Figure 1. Determined by qRT-PCR, The relative expression of MMP13 mRNA increased significantly (Figure 1A). MiR-140-5p increased significantly when compared with control group (Figure 1B). Western blot analysis results showed that IL$1 \beta$ treatment significantly increased NF- $\kappa$ B and NF- $\kappa$ B2 protein level. However, treats with IL-1 $\beta$ effectively suppressed the expression of Smad3 and TGF- $\beta 3$ (Figure 1C).

\section{Smad3 and TGF- $\beta 3$ Downregulated via MiR-140-5p}

To further elucidate the role of miR-140-5p in TMJ-OA like damage, specific miRNA mimics and inhibitors of mouse
miR-140-5p were used for transfection experiment. MCCs were transfected with miR-140-5p mimics, or treated with miR-140-5p inhibitors for $48 \mathrm{~h}$, respectively, followed with or without stimulation of IL-1 $\beta$ for $24 \mathrm{~h}$. As showed in Figure 2, miR-140-5p relative expression level was assessed by qRTPCR. Transfection of miR-140-5p mimics increased miR-140$5 p$ expression significantly (Figure 2A), while inhibitors acted in a converse manner (Figure 2B). Compared with negative control, overexpression of endogenous miR-140-5p significantly reduced Smad3 and TGF- $\beta 3$ protein levels (Figure 2C). Conversely, miR-140-5p inhibitors increased Smad3 and TGF$\beta 3$ protein production, and counteract the stimulation of IL$1 \beta$ markedly (Figure 2D). Consistent with this, the results of immunofluorescence analysis showed the similar fluorescence intensity (Green) changes of Smad3 (Figure 3). When compared with the negative control, overexpression of miR-140-5p reduced the fluorescence intensity of Smad3, especially in the presence of IL-1 $\beta$-induced MCCs. Thus, miR-140-5p contributes to the alteration of Smad3 and TGF- $\beta 3$ expression at protein level.
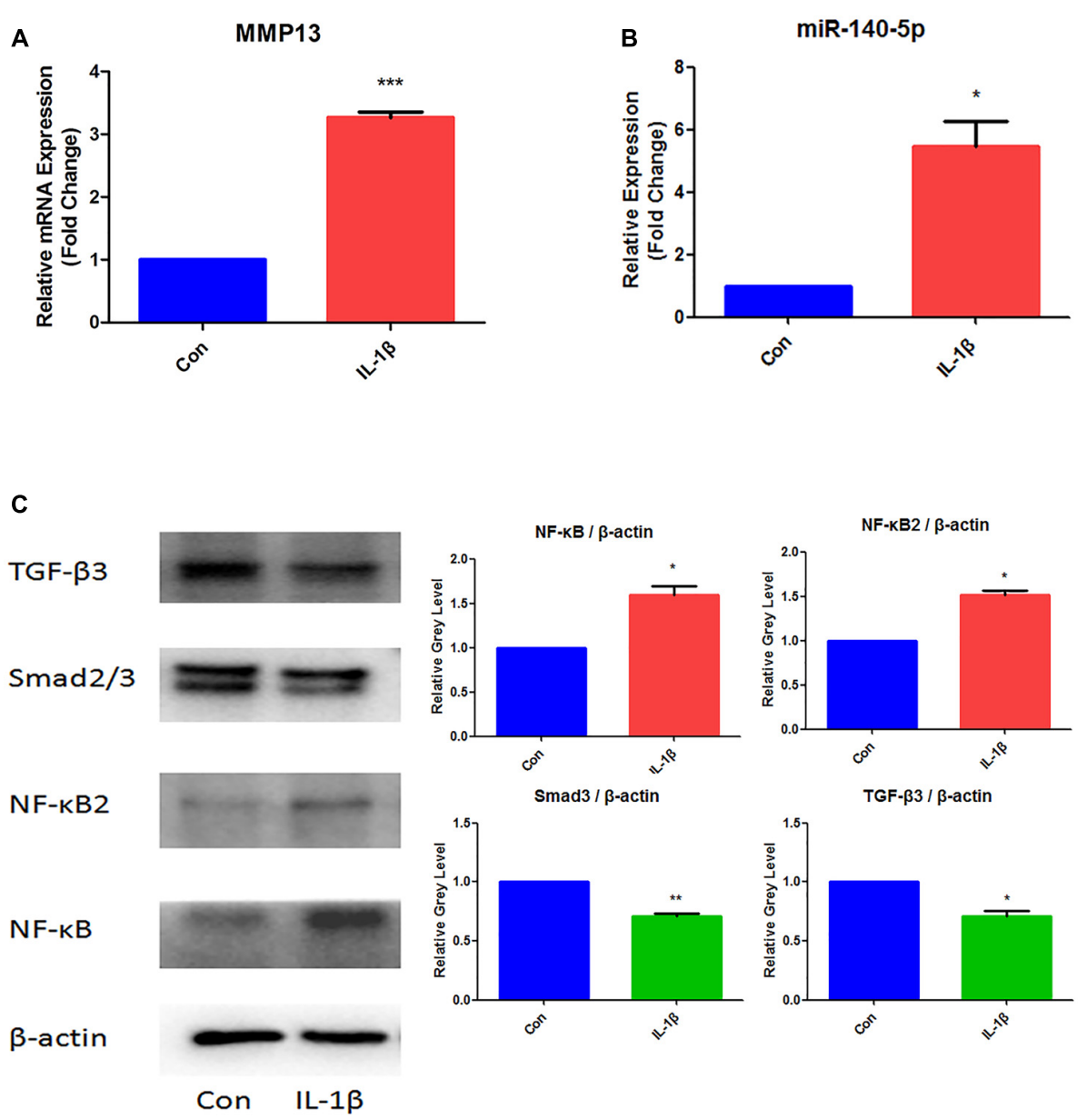

FIGURE 1 | Expression analysis of MMP13, miR-140-5p, NF-кB, NF-кB2, Smad3, and TGF- $\beta 3$ in IL-1 $\beta$-stimulated MCCs. (A) Assessment of MMP13 mRNA relative expression by qRT-PCR, (B) Assessment of miR-140-5p relative gene expression by qRT-PCR, and (C) Evaluation of NF-kB, NF-kB2, Smad3, TGF- $\beta 3$ protein expression. $\beta$-actin and small nuclear RNA U6 were used as controls. Each group repeated three times $(n=3)$. Control group (Con) were without IL-1 $\beta$ stimulation. ${ }^{*} P<0.05$ when compared with Con. ${ }^{* *} P<0.01$ when compared with Con. ${ }^{* * *} P<0.001$ when compared with Con. 
A

miR-140-5p

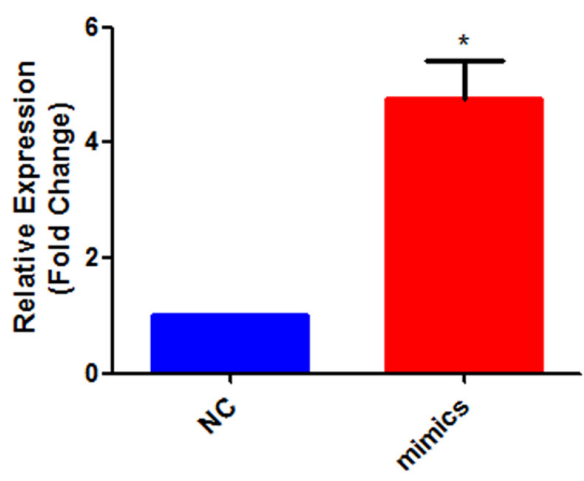

B

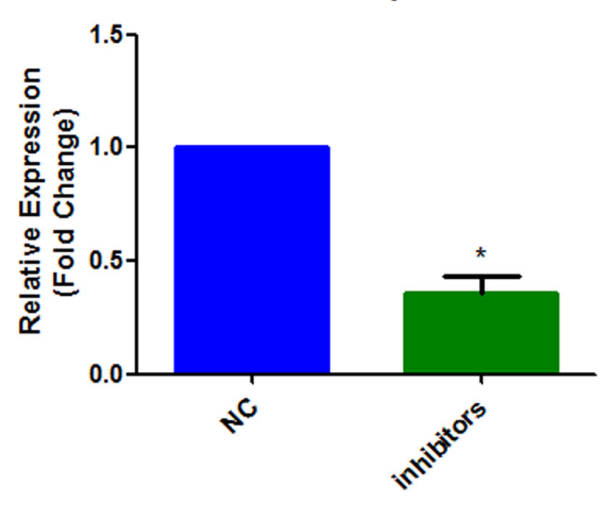

C

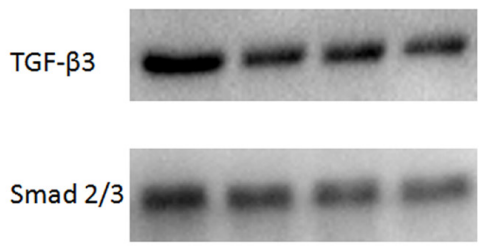

$\beta$-actin
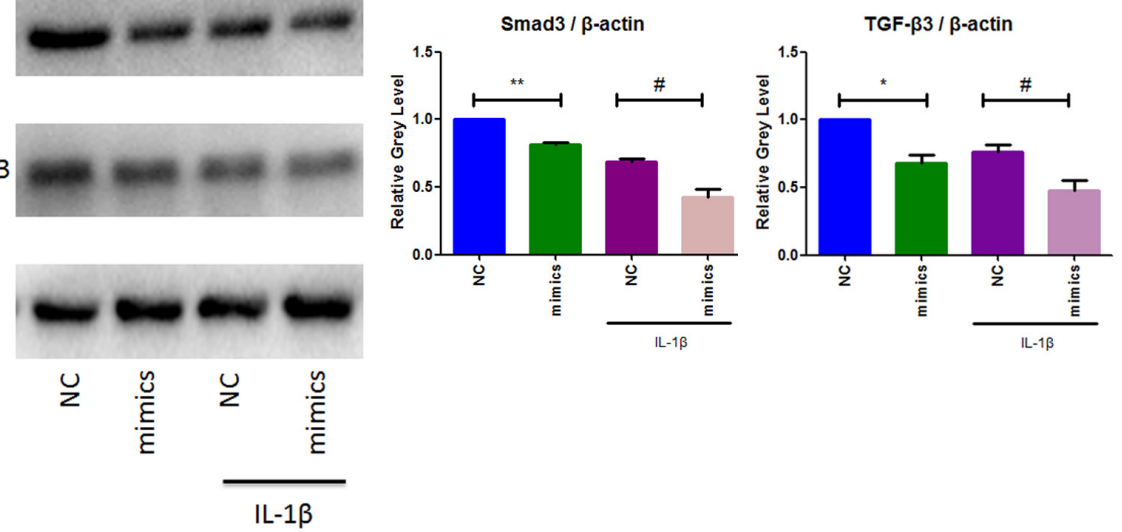

D
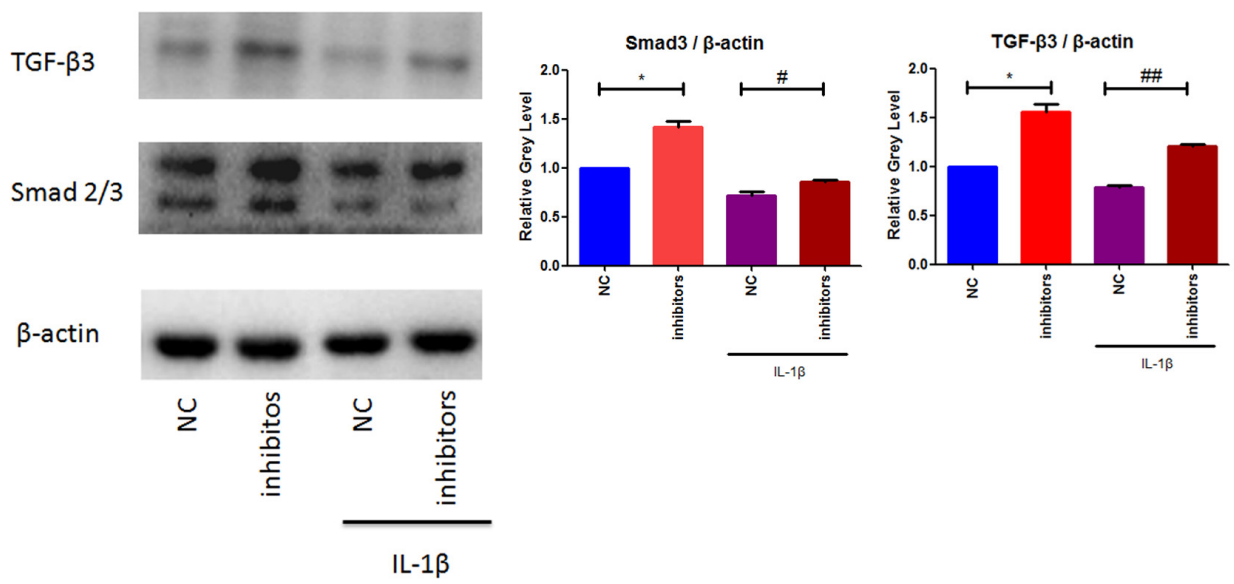

FIGURE 2 | MiR-140-5p mediated Smad3 and TGF- $\beta 3$ in response to IL-1 $\beta$. (A) Evaluation relative microRNAs expression of miR-140-5p in MCCs transfected with mimics, (B) Evaluation relative microRNAs expression of miR-140-5p in MCCs transfected with inhibitors, (C) Evaluation relative protein production of Smad3 and TGF- $\beta 3$ after mimics transfection and stimulation of IL-1 $\beta$ in MCCs, and (D) Evaluation relative protein production of Smad3 and TGF- $\beta 3$ after inhibitors transfection and stimulation of IL-1 $\beta$ in MCCs. $\beta$-actin and small nuclear RNA U6 were used as controls. Each group repeated three times $(n=3)$. ${ }^{*} P<0.05$ when compared with the negative control group (NC). ${ }^{* *} P<0.01$ when compared with NC. ${ }^{\#} P<0.05$ when compared with NC induced by IL-1 $\beta$. ${ }^{\# \#} P<0.01$ when compared with NC induced by IL-1 $\beta$. 

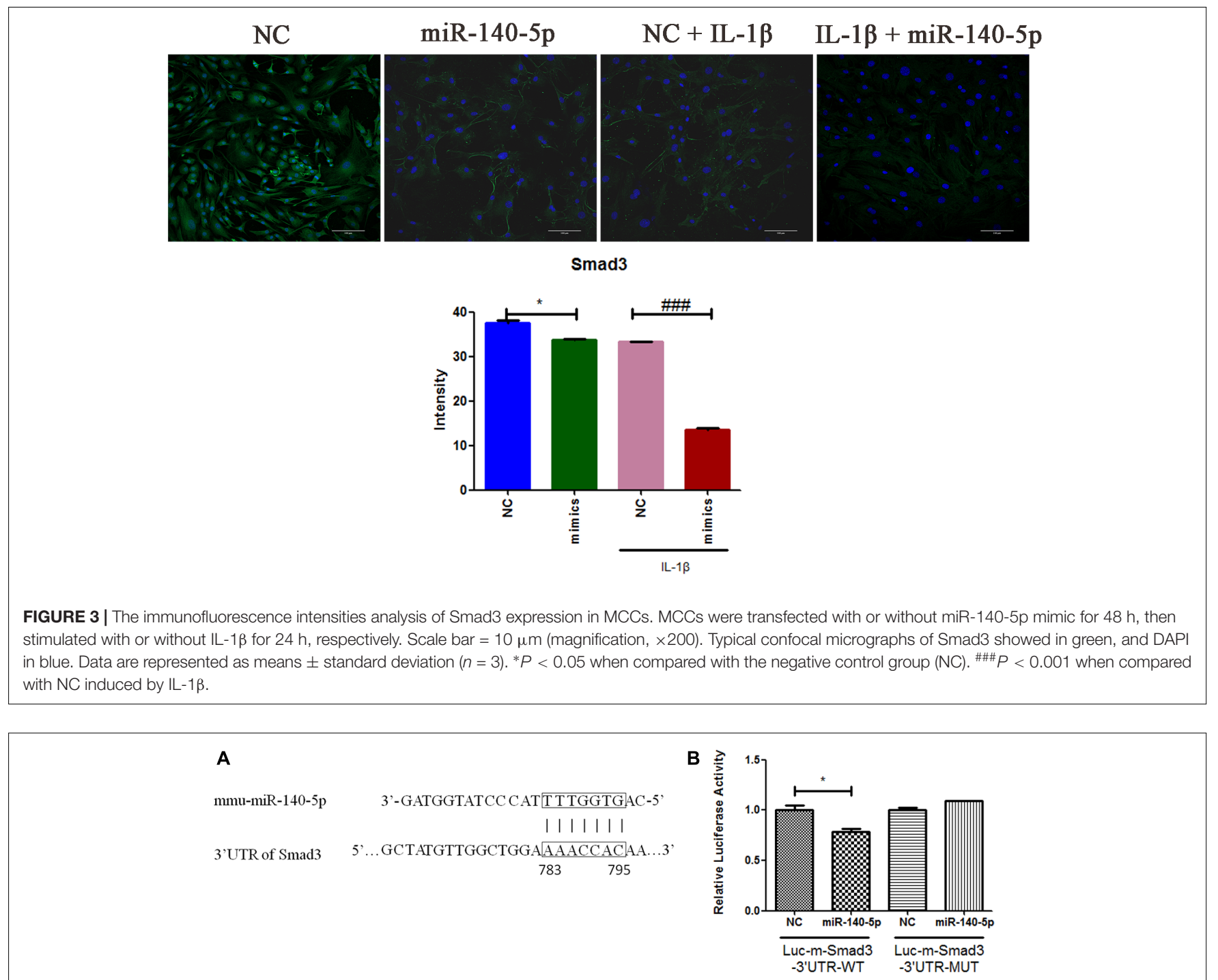

FIGURE 4 | Smad3 mediated by miR-140-5p directly. (A) The predicted binding sites of miR-140-5p to the 3'UTR of Smad3, (B) Analysis of relative luciferase activity. Data were represented as means \pm standard deviation $(n=3)$. ${ }^{*} P<0.05$ vs. negative control group (NC) with Luc-m-Smad3-3'UTR-WT.

\section{MiR-140-5p Directly Inhibits the Expression of Smad3}

To investigate whether miR-140-5p regulates the expression of Smad3 directly, we identified a potential miR-140-5p binding sequence in the $3^{\prime}$-untranslated region ( $3^{\prime}$ UTR) of Smad3 (Figure 4A) by miRNA target prediction software and checked in miRTarBase (Lewis et al., 2005). To further identify the predicted sequence in the $3^{\prime}$ UTRs of Smad3 mRNA interacting with miR$140-5 \mathrm{p}$, cells (293T) were performed transfection with luciferase reporters inserted the target fragment (Luc-m-Smad3-3'UTRWT), or with vectors carrying mutated constructs (Luc-mSmad3-3'UTR-MUT), or miR-140-5p or corresponding negative control (NC), respectively. Cells were harvested after $48 \mathrm{~h}$ transfection. Compared with NC, cells co-transfected with Lucm-Smad3-3'UTR-WT and miR-140-5p showed lower luciferase activity. In contrast, luciferase activity examined no significant change when compared with co-transfection with miR-140-5p and Luc-m-Smad3-3'UTR-MUT, in which the predicted binding sequence to miR-140-5p were mutated (Figure 4B). Together, the data suggested that the miR-140-5p can regulated Smad3 directly.

\section{MiR-140-5p Induced-Downregulation of TGF- $\beta 3$ Is Mediated by Smad3}

To identify the signaling pathway of Smad3 in mediating the downregulation of TGF- $\beta 3$ was induced by miR-140-5p, we utilized small interfering RNA of Smad3 (Si-Smad3) for further transfection experiment. MCCs were co-transfected with or without Si-Smad3 in the presence or absence of miR-140-5p. Cells were harvested after $48 \mathrm{~h}$ transfection. As western blotting results showed in Figure 5, cells transfected with Si-Smad3 exhibit an efficiently reduction of Smad3 and TGF- $\beta 3$. When compared with the miR-140-5p transfection group, cells co-transfected 


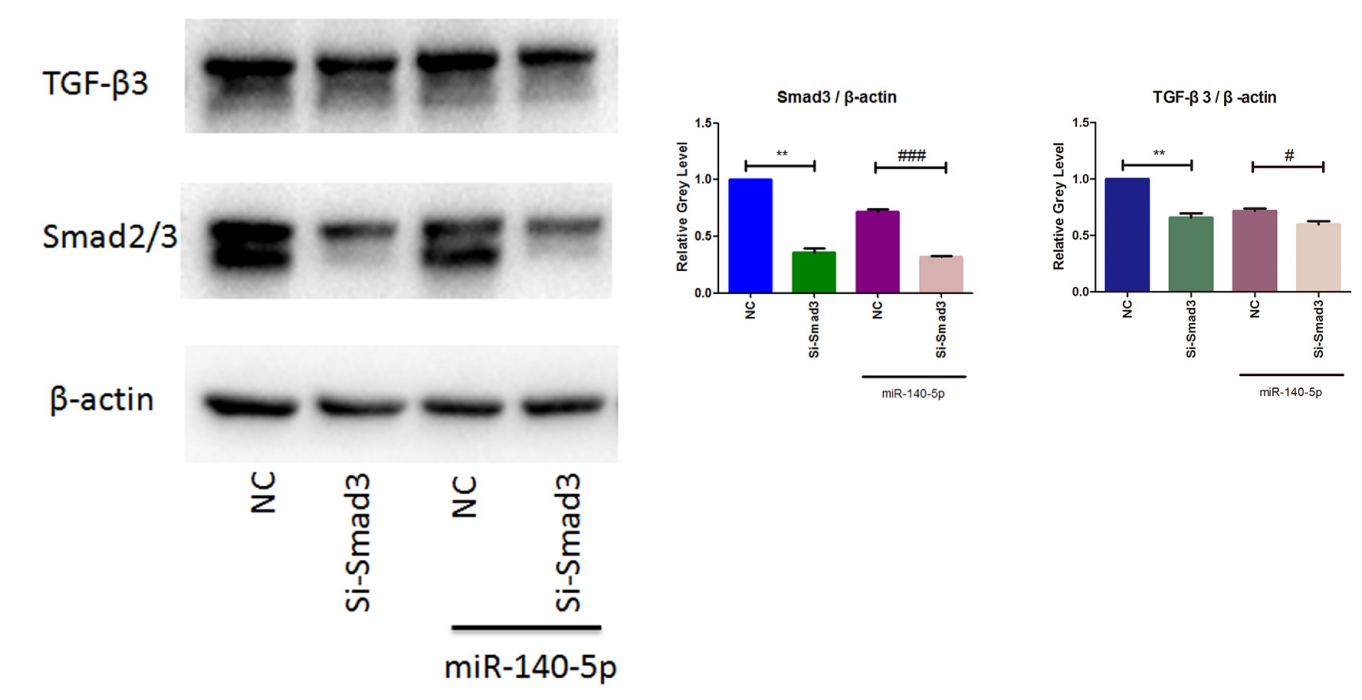

FIGURE 5 | TGF- $\beta 3$ regulated through miR-140-5p/Smad3 signaling. Western blotting analysis of the relative protein expression of Smad3 and TGF- $\beta 3$. Data were represented as means \pm standard deviation $(n=3)$. ${ }^{* *} P<0.01$ vs. negative control group (NC). ${ }^{*} P<0.05$ when compared with NC co-transfected with

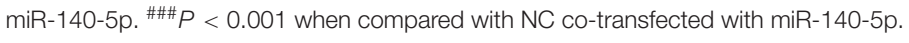

with Si-Smad3 and miR-140-5p blocked Smad3 expression more significantly. What's more, miR-140-5p and Si-Smad3 showed similar effects on downregulation of TGF- $\beta 3$. Interestingly, the group co-transfected with miR-140-5p and Si-Smad3 observed lower TGF- $\beta 3$ expression compared to miR-140-5p group, which suggesting that miR-140-5p induced-downregulation of TGF$\beta 3$ might be more than Smad3 pathway. Together, these results indicate miR-140-5p mediates down-regulation of TGF- $\beta 3$ by Smad3.

\section{DISCUSSION}

As widely accepted, IL-1 $\beta$ is a key mediator in accelerating the activity of MMPs (Ge et al., 2009). MMP13, a representative of MMPs in OA or OA-like changes (de Rooy et al., 2014; Agere et al., 2017; Li et al., 2017; Perotto et al., 2018), reported to be a factor contributes to the early stage development of OA. TMJ-OA is a common disorder in mandibular joint, however, the molecular networks have not been characterized in detail. Due to a limited prediction or therapy for TMJ-OA, the pathogenesis and regulatory mechanism of TMJ-OA urgently need further investigation. NF- $\kappa \mathrm{B}$ is an efficient nuclear factor that accelerate inflammatory reaction (Jiang et al., 2018). The present data showed upregulation of MMP13 and NF- $\kappa$ B in MCCs, which indicated a reliable establishment of TMJ-OA model in vitro.

Previous study reported that TGF- $\beta$ is an essential initiator of chondrogenesis. Inhibition of TGF- $\beta$ caused a reduction of extracellular matrix and resulted in OA (van der Kraan et al., 2012). Smad3 proved to be a key in classic TGF- $\beta$ signal conduction (Yang et al., 2001). Mutant mice of Smad3 developed joint disease like OA (van de Laar et al., 2011). Vertebral and ribs cartilage found TGF- $\beta 3$ highly expressed, particularly in the proliferative and hypertrophic zones (Wang et al., 2014). In the perichondrium, TGF- $\beta 3$ was also expressed at a higher level than other TGF- $\beta$ s (Pelton et al., 1990). TGF- $\beta 3$ enhanced the chondrocyte phenotype and differentiation (Moioli and Mao, 2006; Dahlin et al., 2014). As a result, alteration of TGF- $\beta 3$ may be suitable for the evaluation of OA (Ude et al., 2018). Dysregulation of both miR-140-3p and miR-140-5p were correlated with osteoarthritis (Yin et al., 2017). Recent studies revealed some pathological characteristics of $\mathrm{OA}$, but the exact way of TMJ-OA pathogenesis still need further investigation.

The present study exhibit an increased expression of miR$140-5 p$ was found in MCCs induced by IL-1 $\beta$. MiR-140-5p, originated from miR-140, was reported to be expressed in cartilage particularly. Miyaki et al. (2010) created miR-140-null mice and reported that miR-140 regulates cartilage development and homeostasis. MiR-140 loss was responsible for age-related OA. Wang et al. (2018) found that miR-140-5p/miR-149 effect on chondrocyte metabolism via FUT1. Contradictorily, Swingler et al. (2012) compared human OA cartilage with control cartilage obtained from patients with femoral neck fractures, and found that the expression miR-140-5p was increased in OA cartilage. These contradictory findings may explained by dual-function of miR-140-5p at different development stage of TMJ-OA. Alteration miR-140-5p possibly acted a role like MMP13 in chondrogenesis. Abnormal changes of miR-140-5p may suggest a process of TMJ-OA at early stage. Combined with previous literature, our observation showed a close relationship between miR-140-5p and TGF- $\beta$ pathway. In the present work, we provided evidence that miR-140-5p is a trait of IL- $1 \beta$-induced responsiveness in MCCs, which lead to a reduction of Smad3 and TGF- $\beta 3$ at protein level. Specific miRNA mimics and inhibitors of mmu-miR-140-5p were used for transfection experiment. Overexpression of endogenous miR-140-5p significantly reduced Smad3 and TGF- $\beta 3$ protein levels, but 
miR-140-5p inhibitors acted in a converse manner. Moreover, miR-140-5p inhibitors counteracted the IL$1 \beta$-induced suppression of Smad3 and TGF- $\beta 3$ markedly. Immunofluorescence analysis ensured the conclusion again. Thus, miR-140-5p contributes to the alteration of Smad3 and TGF- $\beta 3$ expression at protein level. The results indicated IL-1 $\beta$ affects Smad3 and TGF- $\beta 3$ through miR-140-5p.

As a key transcription factor in mediating TGF- $\beta$ signaling pathway, Smad3 was observed an inversely relationship to miR140-5-p. Next, we used dual-luciferase constructs and reporter assay to identify whether miR-140-5p inhibits Smad3 expression directly. Dual-luciferase assay demonstrated that miR-140-5p interacted with predicted binding sequence in the $3^{\prime} \mathrm{UTR}$ of Smad3, which consistent with Pais et al.'s (2010) conclusion. Furthermore, Vincenti and Brinckerhoff (2001) reported that TGF- $\beta$ may also suppressed by blocking the expression of Smad4, which found to be a competitive mediator in Smad pathway to control chondrocytes terminal differentiation (van der Kraan et al., 2012).

At last, we provided evidence to demonstrate miR-140$5 p$ regulated the expression of TGF- $\beta 3$ through Smad3. To further investigation, we utilized small interfering RNA of mouse Smad3 (Si-Smad3) to co-transfected with miR-140-5p in MCCs. Compared with negative control, respectively, both of Si-Smad3 and miR-140-5p mimics exhibit an efficiently reduction of TGF$\beta 3$. Interestingly, co-transfection of miR-140-5p mimics and SiSmad3 exhibit a more efficient reduction of TGF- $\beta 3$ protein expression. It suggested that TGF- $\beta 3$ may regulated by miR-140$5 \mathrm{p}$ in multiple ways, including Smad3 pathway. Novel evidences demonstrating that miR-140-3p negatively controlled TGF- $\beta 3$, which proved to be a direct target of miR-140-3p (Fushimi et al., 2018). That's may explained why co-transfection of miR140-5p mimics and Si-Smad3 showed a lower expression of

\section{REFERENCES}

Agere, S. A., Akhtar, N., Watson, J. M., and Ahmed, S. (2017). RANTES/CCL5 induces collagen degradation by activating MMP-1 and MMP-13 expression in human rheumatoid arthritis synovial fibroblasts. Front. Immunol. 8:1341. doi: 10.3389/fimmu.2017.01341

Bouvier, M., and Zimny, M. L. (1987). Effects of mechanical loads on surface morphology of the condylar cartilage of the mandible in rats. Acta Anat. 129, 293-300. doi: 10.1159/000146418

Cordray, F. E. (2016). Articulated dental cast analysis of asymptomatic and symptomatic populations. Int. J. Oral Sci. 8, 126-132. doi: 10.1038/ijos.2015.44

Dahlin, R. L., Ni, M., Meretoja, V. V., Kasper, F. K., and Mikos, A. G. (2014). TGF-beta3-induced chondrogenesis in co-cultures of chondrocytes and mesenchymal stem cells on biodegradable scaffolds. Biomaterials 35, 123-132. doi: 10.1016/j.biomaterials.2013.09.086

de Rooy, D. P., Zhernakova, A., Tsonaka, R., Willemze, A., Kurreeman, B. A., Trynka, G., et al. (2014). A genetic variant in the region of MMP-9 is associated with serum levels and progression of joint damage in rheumatoid arthritis. Ann. Rheum. Dis. 73, 1163-1169. doi: 10.1136/annrheumdis-2013-203375

Duan, D., Li, J., Xiao, E., He, L., Yan, Y., Chen, Y., et al. (2015). Histopathological features of hypertrophic bone mass of temporomandibular joint ankylosis (TMJA): an explanation of pathogenesis of TMJA. J. Craniomaxillofac. Surg. 43, 926-933. doi: 10.1016/j.jcms.2015.03.026

Fushimi, S., Nohno, T., Nagatsuka, H., and Katsuyama, H. (2018). Involvement of miR-140-3p in Wnt3a and TGFbeta3 signaling pathways during osteoblast
TGF- $\beta 3$. At least partially, these results demonstrated miR-140$5 \mathrm{p}$-induced downregulation of TGF- $\beta 3$ was mainly mediated by Smad3.

\section{CONCLUSION}

Taken together, the present study provided evidence that miR$140-5 p$ regulated TMJ-OA pathogenesis through TGF- $\beta / S m a d$ signaling pathway and might be a potential predictive factor for TMJ-OA.

\section{ETHICS STATEMENT}

According to the recommendations of Chinese Academy of Sciences, the protocol of this study was approved by the Animal Experimental Ethics Committee of Kunming Medical University.

\section{AUTHOR CONTRIBUTIONS}

WL and SL designed and developed the research. SZ, HY, CZ, QK, and JD conducted the experiments, and collected and analyzed the data. YX and YD wrote and drafted the manuscript.

\section{FUNDING}

This work was supported by the National Natural Science Foundation of China (81760193 and 81360163), and Graduate Innovation Foundation of Kunming Medical University (2018D004).

differentiation in MC3T3-E1 cells. Genes Cells 23, 517-527. doi: 10.1111/gtc. 12591

Ge, X., Ma, X., Meng, J., Zhang, C., Ma, K., and Zhou, C. (2009). Role of Wnt-5A in interleukin-1beta-induced matrix metalloproteinase expression in rabbit temporomandibular joint condylar chondrocytes. Arthritis Rheum. 60, 2714-2722. doi: 10.1002/art.24779

Goldring, S. R. (2012). Alterations in periarticular bone and cross talk between subchondral bone and articular cartilage in osteoarthritis. Ther. Adv. Musculoskelet. Dis. 4, 249-258. doi: 10.1177/1759720X12437353

Gosset, M., Berenbaum, F., Thirion, S., and Jacques, C. (2008). Primary culture and phenotyping of murine chondrocytes. Nat. Protoc. 3, 1253-1260. doi: 10.1038/ nprot.2008.95

Hashimoto, K., Otero, M., Imagawa, K., de Andres, M. C., Coico, J. M., Roach, H. I., et al. (2013). Regulated transcription of human matrix metalloproteinase 13 (MMP13) and interleukin-1beta (IL1B) genes in chondrocytes depends on methylation of specific proximal promoter CpG sites. J. Biol. Chem. 288, 10061-10072. doi: 10.1074/jbc.M112.421156

Iliopoulos, D., Malizos, K. N., Oikonomou, P., and Tsezou, A. (2008). Integrative microRNA and proteomic approaches identify novel osteoarthritis genes and their collaborative metabolic and inflammatory networks. PLoS One 3:e3740. doi: 10.1371/journal.pone.0003740

Jiang, T., Kai, D., Liu, S., Huang, X., Heng, S., Zhao, J., et al. (2018). Mechanically cartilage-mimicking poly(PCL-PTHF urethane)/collagen nanofibers induce chondrogenesis by blocking NF-kappa B signaling pathway. Biomaterials 178, 281-292. doi: 10.1016/j.biomaterials.2018.06.023 
Kapoor, M., Martel-Pelletier, J., Lajeunesse, D., Pelletier, J. P., and Fahmi, H. (2011). Role of proinflammatory cytokines in the pathophysiology of osteoarthritis. Nat. Rev. Rheumatol. 7, 33-42. doi: 10.1038/nrrheum.2010.196

Lewis, B. P., Burge, C. B., and Bartel, D. P. (2005). Conserved seed pairing, often flanked by adenosines, indicates that thousands of human genes are microRNA targets. Cell 120, 15-20. doi: 10.1016/j.cell.2004.12.035

Li, H., Wang, D., Yuan, Y., and Min, J. (2017). New insights on the MMP-13 regulatory network in the pathogenesis of early osteoarthritis. Arthritis Res. Ther. 19:248. doi: 10.1186/s13075-017$1454-2$

Liu, Y., Wang, Y., Yang, N., Wu, S., Lv, Y., and Xu, L. (2015). In silico analysis of the molecular mechanism of postmenopausal osteoporosis. Mol. Med. Rep. 12, 6584-6590. doi: $10.3892 / \mathrm{mmr} .2015 .4283$

Livak, K. J., and Schmittgen, T. D. (2001). Analysis of relative gene expression data using real-time quantitative PCR and the 2(-Delta Delta C(T)) Method. Methods 25, 402-408. doi: 10.1006/meth.2001.1262

Min, Z., Zhang, R., Yao, J., Jiang, C., Guo, Y., Cong, F., et al. (2015). MicroRNAs associated with osteoarthritis differently expressed in bone matrix gelatin (BMG) rat model. Int. J. Clin. Exp. Med. 8, 1009-1017.

Miyaki, S., Nakasa, T., Otsuki, S., Grogan, S. P., Higashiyama, R., Inoue, A., et al. (2009). MicroRNA-140 is expressed in differentiated human articular chondrocytes and modulates interleukin-1 responses. Arthritis Rheum. 60, 2723-2730. doi: $10.1002 /$ art.24745

Miyaki, S., Sato, T., Inoue, A., Otsuki, S., Ito, Y., Yokoyama, S., et al. (2010). MicroRNA-140 plays dual roles in both cartilage development and homeostasis. Genes Dev. 24, 1173-1185. doi: 10.1101/gad.1915510

Moioli, E. K., and Mao, J. J. (2006). Chondrogenesis of mesenchymal stem cells by controlled delivery of transforming growth factor-beta3. Conf. Proc. Ann. Int. Conf. IEEE Eng. Med. Biol. Soc. 1, 2647-2650. doi: 10.1109/IEMBS.2006.260861

Pais, H., Nicolas, F. E., Soond, S. M., Swingler, T. E., Clark, I. M., Chantry, A., et al. (2010). Analyzing mRNA expression identifies Smad3 as a microRNA140 target regulated only at protein level. RNA 16, 489-494. doi: 10.1261/rna. 1701210

Paniagua, B., Pascal, L., Prieto, J., Vimort, J. B., Gomes, L., Yatabe, M., et al. (2017). "Cevidanes, diagnostic index: an open-source tool to classify TMJ OA condyles," in Proceedings of the SPIE-the International Society for Optical Engineering, Bethesda MD.

Pelton, R. W., Dickinson, M. E., Moses, H. L., and Hogan, B. L. (1990). In situ hybridization analysis of TGF beta 3 RNA expression during mouse development: comparative studies with TGF beta 1 and beta 2. Development $110,609-620$.

Perotto, J. H., Camejo, F. A., Doetzer, A. D., Almeida, L. E., Azevedo, M., Olandoski, M., et al. (2018). Expression of MMP-13 in human temporomandibular joint disc derangement and osteoarthritis. Cranio 36, 161-166. doi: 10.1080/08869634.2017.1315511

Sun, Q., Zhang, L., Xu, T., Ying, J., Xia, B., Jing, H., et al. (2018). Combined use of adipose derived stem cells and TGF-beta3 microspheres promotes articular cartilage regeneration in vivo. Biotech. Histochem. 93, 168-176. doi: 10.1080/ 10520295.2017.1401663

Sun, X., Cao, Z., Zhang, Q., Li, M., Han, L., Li, Y., et al. (2016). Aluminum trichloride inhibits osteoblast mineralization via TGF-beta1/Smad signaling pathway. Chem. Biol. Interact. 244, 9-15. doi: 10.1016/j.cbi.2015.11.027

Swingler, T. E., Wheeler, G., Carmont, V., Elliott, H. R., Barter, M. J., AbuElmagd, M., et al. (2012). The expression and function of microRNAs in chondrogenesis and osteoarthritis. Arthritis Rheum. 64, 1909-1919. doi: 10. 1002/art.34314

Ude, C. C., Shamsul, B. S., Ng, M. H., Chen, H. C., Ohnmar, H., Amaramalar, S. N., et al. (2018). Long-term evaluation of osteoarthritis sheep knee, treated with TGF-beta3 and BMP-6 induced multipotent stem cells. Exp. Gerontol. 104, 43-51. doi: 10.1016/j.exger.2018.01.020

van de Laar, I. M., Oldenburg, R. A., Pals, G., Roos-Hesselink, J. W., de Graaf, B. M., Verhagen, J. M., et al. (2011). Mutations in SMAD3 cause a syndromic form of aortic aneurysms and dissections with early-onset osteoarthritis. Nat. Genet. 43, 121-126. doi: $10.1038 /$ ng.744 van der Kraan, P. M., Goumans, M. J., Blaney Davidson, E., and ten Dijke, P. (2012). Age-dependent alteration of TGF-beta signalling in osteoarthritis. Cell Tissue Res. 347, 257-265. doi: 10.1007/s00441-011$1194-6$

van Rooij, E., Sutherland, L. B., Liu, N., Williams, A. H., McAnally, J., Gerard, R. D., et al. (2006). signature pattern of stress-responsive microRNAs that can evoke cardiac hypertrophy and heart failure. Proc. Natl. Acad. Sci. U.S.A. 103, 18255-18260. doi: 10.1073/pnas.060879 1103

Vincenti, M. P., and Brinckerhoff, C. E. (2001). Early response genes induced in chondrocytes stimulated with the inflammatory cytokine interleukin-1beta. Arthritis Res. 3, 381-388. doi: 10.1186/ar331

Wang, W., Rigueur, D., and Lyons, K. M. (2014). TGFbeta signaling in cartilage development and maintenance. Birth Defects Res. Part C Embryo Today 102, 37-51. doi: 10.1002/bdrc.21058

Wang, Z., Hu, J., Pan, Y., Shan, Y., Jiang, L., Qi, X., et al. (2018). miR-140-5p/miR149 affects chondrocyte proliferation, apoptosis, and autophagy by targeting FUT1 in osteoarthritis. Inflammation 41, 959-971. doi: 10.1007/s10753-0180750-6

Yang, X., Chen, L., Xu, X., Li, C., Huang, C., and Deng, C. X. (2001). TGFbeta/Smad3 signals repress chondrocyte hypertrophic differentiation and are required for maintaining articular cartilage. J. Cell Biol. 153, 35-46. doi: 10. $1083 /$ jcb.153.1.35

Yin, C. M., Suen, W. C., Lin, S., Wu, X. M., Li, G., and Pan, X. H. (2017). Dysregulation of both miR-140-3p and miR-140-5p in synovial fluid correlate with osteoarthritis severity. Bone Joint Res. 6, 612-618. doi: 10.1302/2046-3758. 611.BJR-2017-0090.R1

Zeng, L., Wang, W., Rong, X. F., Zhong, Y., Jia, P., Zhou, G. Q., et al. (2014). Chondroprotective effects and multi-target mechanisms of Icariin in IL-1 beta-induced human SW 1353 chondrosarcoma cells and a rat osteoarthritis model. Int. Immunopharmacol. 18, 175-181. doi: 10.1016/j.intimp.2013. 11.021

Zhang, C., Xu, Q., Xiao, X., Li, W., Kang, Q., Zhang, X., et al. (2018). Prenatal deltamethrin exposure-induced cognitive impairment in offspring is ameliorated by memantine through NMDAR/BDNF signaling in hippocampus. Front. Neurosci. 12:615. doi: 10.3389/fnins.2018. 00615

Zhang, J., Jiao, K., Zhang, M., Zhou, T., Liu, X. D., Yu, S. B., et al. (2013). Occlusal effects on longitudinal bone alterations of the temporomandibular joint. J. Dent. Res. 92, 253-259. doi: 10.1177/0022034512473482

Zhang, J., Liao, L., Zhu, J., Wan, X., Xie, M., Zhang, H., et al. (2018). Osteochondral interface stiffening in mandibular condylar osteoarthritis. J. Dent. Res. 97, 563-570. doi: 10.1177/0022034517748562

Zhang, M., Mani, S. B., He, Y., Hall, A. M., Xu, L., and Li, Y. (2016). Induced superficial chondrocyte death reduces catabolic cartilage damage in murine posttraumatic osteoarthritis. J. Clin. Invest. 126, 2893-2902. doi: 10.1172/ JCI83676

Zhao, Y. P., Zhang, Z. Y., Wu, Y. T., Zhang, W. L., and Ma, X. C. (2011). Investigation of the clinical and radiographic features of osteoarthrosis of the temporomandibular joints in adolescents and young adults. Oral Surg. Oral Med. Oral Pathol. Oral Radiol. Endod. 111, e27-e34. doi: 10.1016/j.tripleo.2010. 09.076

Conflict of Interest Statement: The authors declare that the research was conducted in the absence of any commercial or financial relationships that could be construed as a potential conflict of interest.

Copyright $\odot 2019$ Li, Zhao, Yang, Zhang, Kang, Deng, Xu, Ding and Li. This is an open-access article distributed under the terms of the Creative Commons Attribution License (CC BY). The use, distribution or reproduction in other forums is permitted, provided the original author(s) and the copyright owner(s) are credited and that the original publication in this journal is cited, in accordance with accepted academic practice. No use, distribution or reproduction is permitted which does not comply with these terms. 